\title{
Nonlinear Adaptive Neural Controller for Unstable Aircraft
}

\author{
S. Suresh, ${ }^{*}$ S. N. Omkar, ${ }^{\dagger}$ and V. Mani ${ }^{\ddagger}$ \\ Indian Institute of Science, Bangalore 560 012, India \\ and \\ N. Sundararajan ${ }^{\S}$ \\ Nanyang Technological University, Singapore 63978, Republic of Singapore
}

\begin{abstract}
A model reference indirect adaptive neural control scheme that uses both off-line and online learning strategies is proposed for an unstable nonlinear aircraft controller design. The bounded-input-bounded-output stability requirement for the controller design is circumvented using an off-line, finite interval of time training scheme. The aircraft model is first identified using a neural network with linear filter (also known as time-delayed neural network) with the available input-output data for a finite time interval. The finite time interval is selected such that this time interval is less than the critical time interval for the aircraft from its stability point of view (similar to the time to double). A procedure to select this critical time interval is also presented. For a given reference model and the identified model, the controller neural-network weights are adapted off-line for the same time interval. The off-line trained neural controller ensures the stability and provides the necessary tracking performance for the unstable aircraft. If there is a change in the aircraft dynamics or characteristics, the trained neural identifier and controller are also adapted online. The theoretical results are validated using the simulation studies based on a locally nonlinear longitudinal high-performance fighter aircraft similar to the F-16. The neural controller design proposed is also compared with the feedback error learning neural control strategy in terms of the tracking ability and control efforts for various level flight conditions and fault conditions such as modeling uncertainties and partial control surface loss. We also present the robustness of the aircraft under extreme wind and noise conditions.
\end{abstract}

\section{Introduction}

$\mathbf{O}$ VER the past three decades, adaptive control theory has evolved as a powerful methodology for designing nonlinear feedback controllers for systems with parameter uncertainties. The fundamental issues of adaptive control for linear systems such as selection of controller architecture, assumption on a priori system knowledge, parameterization of adaptive systems, establishment of error models, adaptive law, and analysis of stability have been extensively addressed. These results have been reported in several textbooks dealing with the design and analysis of adaptive systems. ${ }^{1-3}$ However, most practical systems are nonlinear in nature. Adaptive control of such nonlinear systems is therefore an intense area of research. Novel techniques in adaptive control of nonlinear systems are facilitated through advances in geometric nonlinear control theory and, in particular, feedback linearization methods ${ }^{2}$ and backstepping methods. ${ }^{3}$ A key assumption in these methods is that the system nonlinearities are known a priori.

During the past decade, a large amount of research work has been carried out in neural control theory almost independently from adaptive nonlinear control research. ${ }^{4,5}$ Neural networks possess an inherent structure suitable for mapping complex characteristics, learning, and optimization. The feasibility of applying neural-network architectures for identification and control of nonlinear systems was first demonstrated through numerical studies in Ref. 6. In these studies neural networks are mostly used as approximate models for unknown nonlinearities, thus removing the need for a priori knowl-

Received 23 August 2004; revision received 30 November 2004; accepted for publication 25 February 2005. Copyright (C) 2005 by the American Institute of Aeronautics and Astronautics, Inc. All rights reserved. Copies of this paper may be made for personal or internal use, on condition that the copier pay the $\$ 10.00$ per-copy fee to the Copyright Clearance Center, Inc., 222 Rosewood Drive, Danvers, MA 01923; include the code 0731-5090/05 $\$ 10.00$ in correspondence with the CCC.

${ }^{*}$ Research Scholar, Department of Aerospace Engineering. Member AIAA.

${ }^{\dagger}$ Senior Scientific Officer, Department of Aerospace Engineering.

¥Professor, Department of Aerospace Engineering.

${ }^{\S}$ Professor, School of Electrical and Electronic Engineering. Associate Fellow AIAA. edge of system nonlinearities. The nonlinear relationship between the input and output data is represented by the neural-network parameters, also known as weights.

The role of neural network in adaptive neural flight techniques is to learn some underlying relationship between the given inputoutput data and approximate the control law. Different architectures and training schemes have been used for this purpose. ${ }^{6-13}$ Because of their powerful ability of approximating nonlinear functions and control laws, flight controllers incorporating neural network have been extensively studied. A detailed survey on the application of neural networks for nonlinear flight control is presented in Ref. 14. The online learning ability of neural networks is demonstrated by using modeling uncertainties and partial control surface loss in Refs. 15-18. A reconfigurable flight-control algorithm that is trained to distribute control authority among remaining surfaces in a timely fashion without explicit knowledge of a given failure condition is reported in Refs. 12, 19, and 20. A complete survey of the relevant literature is given in Ref. 21.

Among the various neural-network-based flight-control schemes, the feedback error learning scheme is quite common. In the feedback-error learning-neural-control (FENC) scheme, the control architecture uses a conventional controller in the inner loop to stabilize the system dynamics, and the neural controller acts as an aid to the conventional controller for compensating the nonlinearity. Under severe modeling uncertainties, fault conditions, and time-varying nonlinear dynamics of the plant, the neural network is adapted online to ensure better tracking ability, provided the basic conventional controller satisfies bounded-input-bounded-output (BIBO) stability requirement. Because the conventional controller is not designed for the new conditions, the control effort required by the FENC scheme is usually high when compared to the adaptive neural controllers. Recently ${ }^{22}$ different conventional and neural controllers were designed for various fault and nominal conditions. A switching technique based on performance measure is used to select the appropriate controllers. But identifying the fault conditions and switching to appropriate controller is often difficult. To overcome these difficulties, a model reference indirect adaptive neural control (MRIANC) for unstable plants that incorporates a new scheme with both off-line and online learning is presented. 